\title{
Médiévales
}

Langues, Textes, Histoire

49 | automne 2005

La paroisse, genèse d'une forme territoriale

\section{Le prince des cuisiniers et le cuisinier des princes : nouveaux documents sur maestro Martino}

The Prince of Cooks and the Cook of Princes: New Documents Concerning Maestro Martino

Bruno Laurioux

\section{(2) OpenEdition}

Journals

Édition électronique

URL : https://journals.openedition.org/medievales/1236

DOI : 10.4000/medievales. 1236

ISSN : $1777-5892$

Éditeur

Presses universitaires de Vincennes

Édition imprimée

Date de publication : 1 décembre 2005

Pagination : 141-154

ISBN : 2-84292-177-1

ISSN : 0751-2708

\section{Référence électronique}

Bruno Laurioux, «Le prince des cuisiniers et le cuisinier des princes : nouveaux documents sur maestro Martino », Médiévales [En ligne], 49 | automne 2005, mis en ligne le 02 novembre 2010 consulté le 23 avril 2022. URL : http://journals.openedition.org/medievales/1236 ; DOI : https:// doi.org/10.4000/medievales. 1236

Ce document a été généré automatiquement le 23 avril 2022.

Tous droits réservés 


\section{Le prince des cuisiniers et le cuisinier des princes : nouveaux documents sur maestro Martino}

The Prince of Cooks and the Cook of Princes: New Documents Concerning

Maestro Martino

Bruno Laurioux

1 Le portrait que, dans son De honesta voluptate, Platina dresse du cuisinier est très flatteur :

Du cuisinier. Que l'on ait un cuisinier instruit par l'art et une longue expérience, patient dans son travail et qui recherche avant tout à être loué pour celui-ci. Qu'il soit dépourvu de toute malpropreté et saleté, qu'il connaisse de manière appropriée la puissance et la nature des viandes, poissons et légumes, afin qu'il distingue ce que l'on doit faire rôtir, bouillir ou frire. Qu'il soit très actif pour discerner par le goût ce qui est trop salé et ce qui est fade. Qu'il soit si possible entièrement semblable en tout au Néo-Comasque, le prince des cuisiniers de notre temps, dont j'ai reçu la méthode pour préparer les mets. Qu'il ne soit ni goinfre ni vorace, comme l'était Marisius Gallus, de manière à ce qu'il ne prenne ni ne dévore ce que son maitre doit manger ${ }^{1}$.

2 Si la dévalorisation médiévale du travailleur manuel confronté à la graisse des cuisines demeure en arrière-plan de ce passage, il est tentant de relier le cuisinier de Platina au personnage à la fois érudit et bavard, ridicule et expert, dont la littérature humaniste est friande. À l'évidence, il manifeste également le nouveau statut qu'a atteint l'art culinaire aux $\mathrm{XIV}^{\mathrm{e}}$ et $\mathrm{XV}^{\mathrm{e}}$ siècles : celui d'une pratique digne d'être mise par écrit et dont les maîtres jouissent d'une respectabilité professionnelle et sociale ${ }^{2}$. Or, l'humaniste désigne explicitement comme parangon de ce cuisinier parfait - on a envie d'écrire de ce nouveau cuisinier - un certain Martino ${ }^{3}$, qui s'est révélé être un individu parfaitement réel. Des recherches actives ont même permis de découvrir plusieurs versions du recueil de recettes qu'il avait composé et dont Platina s'était tant inspiré : bénéficiant du croissant intérêt du grand public pour la cuisine médiévale, ce Libro de arte coquinaria est ainsi devenu en Italie une véritable icône, et Martino le porte- 
drapeau d'un nationalisme culinaire qui n'est pas sans rapport avec le nationalisme politique.

Le modeste objectif de cette note est de corriger le portrait que l'on peut dresser du cuisinier, à la lumière de récentes découvertes dans les Archives romaines ${ }^{4}$. Au final, Maestro Martino apparaît comme le symbole d'un nouveau type de cuisinier, partagé entre le service de l'Église et celui de l'État (princier), et ceci beaucoup plus qu'on ne l'imaginait il y a une cinquantaine d'années.

\section{Maestro Martino : un état des lieux}

Depuis sa redécouverte par Joseph Dommers Vehling durant les années $1930^{5}$, la figure de Maestro Martino a acquis une consistance singulière. Ce qu'on savait juste après la Seconde Guerre mondiale se résumait en peu de choses, tirées du seul manuscrit de son livre de cuisine (le Libro de arte coquinaria) que l'on connaissait alors (Washington, Library of Congress, Rare Books 153, abrégé en $W$ ) : ce cuisinier d'exception, admiré par Platina, avait été " jadis " (olim) au service d'un "camérier et patriarche d'Aquilée " ${ }^{6}$ qui n'est évidemment autre que Ludovico Trevisan ${ }^{7}$. C'est d'abord le repérage de nouveaux témoins manuscrits qui a permis de compléter très significativement la carrière de Martino ${ }^{8}$.

Le premier événement - malheureusement passé inaperçu des (rares) spécialistes de l'époque - a été la brève réapparition au milieu des années $1970^{\circ} \mathrm{d}$ 'un manuscrit resté constamment en mains privées et qui avait fait partie, au xIX $x^{e}$ siècle, des prestigieuses collections d'Ambroise Firmin-Didot et de Jérôme Pichon ${ }^{10}$. Redisparu depuis dans la nature ${ }^{11}$, ce manuscrit $(=F)$ exceptionnellement orné pour un livre de cuisine, possède le grand intérêt de transmettre un texte manifestement écrit alors que Martino était encore au service du cardinal d'Aquilée ${ }^{12}$.

C'est surtout la découverte d'un manuscrit nettement moins luxueux, conservé à la Biblioteca civica de Riva del Garda $(=R)^{13}$, qui a fait progresser d'un coup notre connaissance de Maestro Martino : les deux titres qu'il comporte ${ }^{14}$ révèlent en effet son nom (de' Rossi) ${ }^{15}$, son lieu de naissance (la vallée tessinoise du Blenio ou Brenno, en Suisse actuelle) et un nouveau patron (le fameux capitaine Gian Giacomo Trivulzio). Il y a tout lieu de croire que l'épisode Trivulzio se situe après celui de Trevisan : la mort de ce dernier a lieu, rappelons-le, en mars 1465 ; or, la carrière du condottiere ne commence pas avant $1467{ }^{16}$. D'autre part, l'écriture trahit une copie tardive et le texte manifeste un réaménagement d'ampleur de l'œuvre (avec de nouvelles recettes et des regroupements) et ne peut donc nullement être considéré comme le noyau primitif du recueil. Ce qu'est venue confirmer, tout récemment, la découverte de feuillets enlevés à ce manuscrit : l'un des repas dont ils donnent le menu date en effet de $1501{ }^{17}$. Comme il s'agit du festin de noces de Niccolò Trivulzio, le fils du condottiere, il n'est pas exclu que ce soit encore Martino qui l'ait préparé ${ }^{18}$. Le jeune époux (il est né en 1479) y est qualifié de comte de Mesocco, localité qui se situe dans une vallée voisine de celle du Brenno : Maestro Martino serait-il ainsi revenu à ses origines?

En accompagnement du facsimilé du manuscrit de Riva del Garda, Giuseppe Chiesi a analysé quelques documents pouvant concerner Maestro Martino ${ }^{19}$ et qui ajoutent à sa carrière un nouveau volet, très précoce et milanais celui-là. Il n'est pas sûr que les huit actes ou lettres rassemblés par Chiesi s'appliquent bien à la même personne. Les plus 
incontestables sont : $1^{\circ}$ une "licentia eundi usque in vallem Belegni » délivrée par le duc Francesco Sforza, le 27 juin 1457, à «Magistro Martino de dicta valle, coquo camerariorum ", autorisation valable pour le mois suivant pour se rendre dans la vallée en question ; $2^{\circ}$ un mandement de la duchesse Bianca Maria, du 8 janvier 1461, destiné à ce que «Rubeus de Blegnio, coquus noster " soit réglé de ses créances. Les autres documents, qui s'étagent de 1442 à 1462, mentionnent selon les cas un «Rubeum de Blegnio, famulum nostrum ", un "Rosso quale serve qui alla corte nostra » voire un simple "Rosso", qui ne sont pas forcément des cuisiniers, même s'ils ont toujours affaire à la même vallée - où il ne doit pas y avoir qu'un seul Rossi ${ }^{20}$ ! La présence de Maestro Martino à la cour de Milan dans les années 1461-1462 a été récemment contestée par Claudio Benporat qui interprète coquus camerariorum comme « cuisinier du camérier ", c'est-à-dire de Ludovico Trevisan ${ }^{21}$. Outre le fait que camerariorum n'est pas camerarii, on doit remarquer que l'acte invoqué par Benporat est de 1461 et non de 1467 comme il l'écrit - ce qui, d'ailleurs, eût rendu tout à fait impossible l'allusion à Trevisan. Rien ne s'oppose donc à ce que Martino soit entré au service du patriarche d'Aquilée à partir, par exemple, de 1462.

\section{Maestro Martino, cuisinier des papes}

Grâce à d'heureuses découvertes ${ }^{22}$, il est possible de compléter le versant romain de la carrière de Maestro Martino en suggérant qu'il fut, de 1464 à 1484 au moins, cuisinier secret des papes Paul II, puis Sixte IV. On trouvera ci-après les documents sur lesquels j'appuie mes dires. Ils n'ont pas la prétention d'être exhaustifs et l'occasion se présenterait sans doute d'en trouver des compléments dans d'autres types de comptabilités ainsi que dans des séries archivistiques toutes différentes. Tels quels, ils attestent la position dont Martino jouissait à l'intérieur de l'hôtel pontifical, ils donnent une idée des activités qui étaient les siennes et laissent entrevoir la manière dont il était rétribué.

Contrairement à Jean de Bockenheim ${ }^{23}$, Maestro Martino exerçait son art pour la personne même du pape (documents ci-dessous, $n^{\text {os }} 1$ et 6 à 15 ) ${ }^{24}$. Bien qu'il n'en soit pas l'unique « cuisinier secret » ${ }^{25}$, il semble avoir bénéficié d'une certaine prééminence sur ses collègues ${ }^{26}:$ n'a-t-il pas dirigé la brigade occupée à préparer la collation de San Marco du vendredi 13 février 1466 (doc. 4 et 5), ce véritable coup politique grâce auquel Paul II voulut marquer son pouvoir et souhaitait s'attirer les bonnes grâces du peuple romain? C'est lui qui se chargera de distribuer entre «ses » cuisiniers le salaire de 20 florins qu'il reçoit quelques jours plus tard. C'est lui encore qui, une vingtaine d'années plus tard, avance l'argent pour assurer l'approvisionnement régulier en œufs frais dont la table pontificale a constamment besoin ( $\mathrm{n}^{\circ \mathrm{s}} 8,10,12-13$ et 15$)$. Il veille aussi au remplacement des récipients de cuisine et au réassort en parmesan $\left(n^{\circ s} 9,11,14\right)$, commande si nécessaire du nouveau matériel ${ }^{27}$.

Sur Maestro Martino cuisinier secret du pape subsistent cependant bien des doutes. Si un homme portant ce nom a bien exercé ces fonctions auprès de Paul II et de Sixte IV, est-il le même que notre Martino, c'est-à-dire l'auteur du Libro de arte coquinaria? Les risques d'homonymie pour un nom aussi courant que Martin ne sont pas à négliger : outre le possible cuisinier du commun, signalons, dans le compte même de la collation de 1466, un "Martino Pergamascho ", c'est-à-dire de Bergame, qui exerce l'honorable profession de maçon ${ }^{28}$. Que Maestro Martino soit déjà actif en novembre 1464, alors 
que le patriarche d'Aquilée n'est pas encore mort, ne pose en revanche guère de problème : débaucher les bons cuisiniers est une pratique courante de l'époque. Plutôt que de débauchage, il vaudrait mieux parler au reste de partage car, si l'on a raison de supposer que le patriarche se déplaça au palais pour y mourir, on ne voit pas pourquoi son cuisinier ne l'y aurait pas suivi : le jardin dont il détenait les clés ${ }^{29}$ pourrait avoir été, finalement, celui du cardinal. Voilà pour l'amont. Vers l'aval, la transition fait encore moins difficulté. Claudia Märtl n'a pas trouvé trace de Maestro Martino après 1484 et il y a tout lieu de la croire - et d'en tirer la conclusion que notre homme quitta le service pontifical après la mort de Sixte IV. Passa-t-il directement à celui de Trivulzio ? Je l'ignore.

11 Mais la principale objection à ce que l'auteur du Libro de arte coquinaria ait été au service du pape réside dans le simple fait qu'aucun des manuscrits ayant transmis son œuvre ne fait mention d'un épisode qui a duré tout de même vingt ans. Or, Maestro Martino, dans les successifs réaménagements qu'il a imposés à son recueil, a l'habitude - fort répandue à l'époque - de rendre hommage à son maître du moment : Ludovico Trevisan pour le manuscrit $F$, Gian Giacomo Trivulzio pour R. Pis, $W$ présente Martino, on s'en souvient, comme ayant été "jadis» au service du Patriarche, ce qui n'aurait pas manqué de paraître injurieux pour le successeur de Trevisan, c'est-à-dire le pape en personne. Il se peut toutefois qu'on ait là le résultat de l'intervention isolée d'un copiste ayant su que le cardinal d'Aquilée était mort.

Claudia Märtl a proposé de voir dans le manuscrit $F$ une copie de dédicace (avortée) à Paul II ${ }^{30}$ : cet exemplaire comporte, au bas de sa magnifique page de titre, un écusson qui n'a pas été complété par des armes. Que ces armes virtuelles eussent été celles du pape Barbo me parait tout à fait impossible : puisque Martino était passé à son service, pourquoi, encore une fois, aurait-il laissé le nom de son ancien maître sur le manuscrit qu'il comptait offrir au nouveau? Je crois beaucoup plus plausible que la mort du cardinal ait empêché la peinture des armes. En revanche, lorsque Claudia Märtl voit en Platina l'artisan de ce qu'elle présente (à tort) comme un ratage, elle n'est peut-être pas très loin de la vérité ${ }^{31}$.

Car s'il y a bien une personne qui ne souhaitait pas que le nom de Paul II figurât sur un livre, c'est bien Bartolomeo Platina. Or, le manuscrit $F$ a été copié dans une écriture qui ressemble de très près à celle de Bartolomeo Sanvito ${ }^{32}$, le scribe attitré de l'Académie romaine. Certes, celui-ci a surtout exercé son art raffiné de l'humanistique au profit de la seconde Académie qui fleurissait sous Sixte IV, mais Claudia Märtl a retrouvé la trace d'un de ses passages à Rome entre la fin de l'année 1464 et le début de $1465^{33}$. Quand bien même d'ailleurs le manuscrit eût été copié plus tard, Platina n'aurait pas été fâché d'y conserver le nom d'un vieil adversaire de Pietro Barbo, chez lequel il avait pu faire la connaissance de Maestro Martino, plutôt que de lui substituer le nom du pape honni qui avait fini par débaucher le cuisinier de son cœur.

À la lumière de ces nouvelles données, il faut convenir que les occasions de rencontre entre Platina et Martino n'ont pas manqué. Milan n'est a priori pas à exclure, si l'on veut bien se rappeler que Platina servit sous les ordres de Francesco Sforza ${ }^{34}$; mais c'était au début des années $1440^{35}$, à une époque où rien n'atteste l'activité du cuisinier Maestro Martino soit à la cour qui était encore celle des Visconti ${ }^{36}$, soit auprès du mercenaire qui allait bientôt prendre leur place. Si Crémone (près duquel Platina est né) se trouve bien en territoire milanais et si les relations suivies entre la cour des Gonzaga et celle des Sforza ont pu amener un jour ou l'autre l'humaniste à entrer en 
contact avec le cuisinier de la duchesse de Milan ${ }^{37}$, admettons que les chances de rencontre sont restées faibles jusqu'au début des années 1460, moment où Martino est probablement entré au service du cardinal Trevisan. Elles sont bien entendu devenues inexistantes à compter du décès de Platina, en septembre 1481.

Mais à Rome, le séjour de Maestro Martino a finalement duré vingt ans de plus que ce que l'on imaginait. L'humaniste, que ses amis accusaient de rechercher avidement les bonnes tables ${ }^{38}$, a dû vouloir fréquenter celle qui pouvait apparaître comme la meilleure de Rome au début des années 1460, c'est-à-dire celle du cardinal Trevisan. Il a certainement suivi son cher cardinal Gonzaga lorsque celui-ci venait rendre visite au patriarche dans sa fraîche villa d'Albano. Sous Paul II, il a été à plusieurs reprises l'hôte forcé des prisons du pape - mais sans doute pas invité à sa table ${ }^{39}$. C'est entre ses deux longs séjours au Château-Saint-Ange que Platina a probablement mis la dernière main à son De honesta voluptate : Martino était à l'époque au sommet de sa carrière et peut-être de son art - même s'il exerçait celui-ci au profit du pire ennemi de l'humaniste.

\section{Mentions de Maestro Martino dans les documents comptables de la papauté 40}

1. Comptes de menues dépenses (1464.XI.29)

«Item per do chiave feze fare $\mathrm{m}$ [aestr]o martin de la cozina secreta, prima a la porta de lorto e laltra a [non complété] 9 bol. »

ASR, Camerale I, 1479, fo $8 \mathrm{r}^{\circ}$

2. Comptes de menues dépenses (1464.XII.05)

« Contado a M[aestro] Martin de la cozina commune chel pago per la seratura cum la chiave ad $\mathrm{I}^{\circ}$. armario de la cozina et per $\mathrm{I}^{\mathrm{a}}$. chiave a la porta de la cozina che la sua era rotta et per do candelieri de ferro per la dita cozina. »

ASR, Camerale I, 1479, fo $9 \mathrm{r}^{\circ}$

3. Comptes de menues dépenses, compte spécial de la collation du 17.II.1466, placé après les relevés journaliers de tout le mois (1466.II)

"Spese fece per la collatione generalle fatta in piacca de San Marco [...] Item per 8 forme de legne da fare le raviole fece fare m[aestr]o Martino duc. [0] bol. 21 »

ASR, Camerale I, 1479, fo $116 \mathrm{r}^{\circ}$

4. Mandat de paiement de la Chambre Apostolique (1466.II.26) 
«<en marge> Pro coquis collationis carnisprevii ». <texte> « Marcus ${ }^{41}$ etc. Solvi faciatis Coquis qui pararunt collationem generalem factam Civibus Romanis apud Sanctummarcum die veneris ante carnisprivium proxime preteritis Seu magistro Martino Coquo et familiari Sanctum domini nostri pape eorum asserto procuratori pro eis florenti auri de Camera viginti pro eorum salario et mercede eorum laboris recipienti parationis collationis predicte quos in vestris computis admictemus. Datis ut supra. [i.e. 26.II.1466]»

ASR, Camerale I, 839, fo $163 \mathrm{r}^{\circ}$

5. Introitus et Exitus (1466.III.07)

" < marge gauche> Pro cochis. Doc. B. R. ${ }^{42}$ Doc. A. de Forl. <texte> Dicta die de mandato et per manus nostris prefatus dominus commissariis thesaurarii dedit et solvit Martino Cocho in palatio apostolico flor. similes viginti solvendos per eum nonnullis aliis cochis qui pararunt collationem in die veneris ante carnis privium factam civibus romanis, ut apparet per mandatum factum.xxvj. febr[uariis] provisione predicta... proxime predicti. $<$ marge droite> flor. XX »

ASV, Cam. Ap., Intr. et Ex., 465, fo $147 r^{\circ}$.

Le même paiement est consigné dans le registre jumeau Intr. et Ex. 466, fo 148ro. Pour cette année, nous ne possédons pas l'exemplaire du dépositaire, a priori plus précis.

6. Compte du cubiculaire (1469.IV.02)

« <en marge> pro cocis secretis. Eodem die S.d.n. dedit per manus domni Petri cubicularii magistro Martino coco secreto duc. largos decem et magistro Petro coco secreto duc. largos octo et Iohanni in coquina secreta duc. largos sex : duc. 24. »

ASR, Camerale I, 1481, fo $9 \mathrm{r}^{\circ}$

7. Compte du cubiculaire (1469.XII.08)

« <en marge> pro Magistro Martino coco. Eadem die S.d.n. per manus domini Petri cubicularii largitus est magistro Martino coco secreto duc. papales octo : duc. 8. »

ASR, Camerale I, 1481, fo $29 \mathrm{r}^{\circ} 43$

8. Comptes de menues dépenses (1483.XI.14)

«pro ovis recentibus 124 receptis in coquina secreta per Magistrum Martinum a di 29 septembris usque ad presentem diem a Pichinino de Treve : bol. 60. »

ASR, Camerale, I, 1483, fo $79 \mathrm{v}^{\circ}$

9. Comptes de menues dépenses (1483.XI.17)

" pro Magistro Martino coco secreto quando ivit ad Ostiam videlicet per expensas ipsius in itinere : bol. 14. Item pro caseo parmisano empto per ipsum magistrum Martinum :

bol. 107,5.

ASR, Camerale I, 1483, fo $81 \mathrm{r}^{\circ}$

10. Comptes de menues dépenses (1483.XII.17)

" pro ovibus recentibus 263 ad bol. 1 pro duobus receptis per Magistrum Martinum cocum secretum a die 14 novembris usque ad diem presentem : duc. 1 bol. 56."

ASR, Camerale I, 1483, fo $92 \mathrm{v}^{\circ}$ 
11. Comptes de menues dépenses (1484.I.03)

« pro pignettis emptis per Magistrum Martinum pro coquina secreta de mense novembris duc. 1 bol. 15. Pro caseo empto per dictum Magistrum Martinum bol. 5. »

ASR, Camerale I, $1483, f^{\circ} 100 v^{\circ}$

12. Comptes de menues dépenses (1484.I.06)

« pro ovibus recentibus 130 receptis per Magistrum Martinum cocum secretum a die 18 decembris usque ad diem presentem : bol. 64. »

ASR, Camerale I, 1483, fo $102 \mathrm{r}^{\circ}$

13. Comptes de menues dépenses (1484.V.19)

« pro ovibus recentibus 832 ad bol. 1 pro tribus ovis receptis in coquina secretas et emptis per Magistrum Martinum cocum secretum a die 23 aprilis usque ad presentem : duc. 3 bol. 52. Pro pistonis emptis per Magistrum Martinum cocum secretum : bol. 15. »

ASR, Camerale I, 1483, fo $^{\circ} 158 \mathrm{v}^{\circ}$

14. Comptes de menues dépenses (1484.VII.6)

" pro pignattibus pro secreto Iamdiu receptis et emptis per Magistrum Martinum cocum secretum : duc. 1 bol. 30.»

ASR, Camerale I, $1483, f^{\circ} 178 \mathrm{v}^{\circ}$

15. Comptes de menues dépenses (1484.VII.20)

" pro ovibus recentibus 742 ad bol. 1 pro duobus ovis receptis per Magistrum Martinum cocum secretum a die 19 maii usque ad diem presentem : duc. 1 bol. 56. »

ASR, Camerale I, 1483, fo $183 \mathrm{r}^{\circ}$

\section{NOTES}

1. "Coquum habeat arte et longa experientia doctum, patientem laboris et qui laudari in ea re maxime cupiat. Careat is omni squalore ac spurcitia, cognoscat apposite carnium piscium holerum vim atque naturam ut quid assum quid elixum quid frictum fieri debeat deprehendat. Caleat gustu decernere quae salita nimis et quae fatua sunt. Novicomensi nostra aetate coquorum principi, et a quo obsoniorum conficiendorum rationem accepi, sit omnino, si fieri potest, persimilis. Non sit gulosus et edax, ut Marisius Gallus, ne quod dominum edere oportet ipse intercipiat et devoret » (Platina, De honesta voluptate, M. E. Milham éd., On Right Pleasure and Good Health, Tempe [Az.], 1998 [MRTS, 168], I, 11, p. 119 [désormais abrégé en DHV]). Au contraire de Mary Ella Milham («the Frenchman Marisius » : ibid.), j'ai interprété Gallus comme un patronyme plutôt que comme l'indication d'une origine géographique.

2. Le parallèle entre les qualités que Platina exige du bon cuisinier et le prologue du Fait de cuisine du savoyard Maître Chiquart (1420) est frappant: T. Scully, " "Aucune science de l'art de cuysinierie et de cuysine" : Chiquart's Du fait de cuisine », Food and Foodways, 2-2, 1987, p. 199-214.

3. Cela ressort d'une autre notice du De honesta voluptate : «Quel cuisinier - ô dieux immortels !tu apportes avec mon [ami] Martino de Côme, dont je tiens une grande partie de ce que j'écris » («Quem coquum, dii immortales, Martino meo Comensi conferes, a quo haec scribo magna ex parte sunt habita ", DHV, VI, 41, Cibaria alba). 
4. Il complète donc le bilan dressé il y a neuf ans dans B. Laurioux, « I libri di cucina italiani alla fine del Medioevo : un nuovo bilancio », Archivio Storico Italiano, 154, 1996, p. 33-58. Voir aussi O. Redon, "Les métiers de cuisinier", dans Ceti, modelli, comportamento nella società medievale (Secoli XIII-metà XIV), Atti del diciassettesimo Convegno Internazionale di studi tenuto a Pistoia nei giorni 16-19 maggio 1997, Pistoia, 2001, p. 273-290.

5. J. D. Vehling, "Martino and Platina Exponents of Renaissance Cookery ", Hotel Bulletin and Nation's Chef, octobre 1932, p. 192-193 et surtout Platina and the Rebirth of Man, Chicago, 1941.

6. «Libro de arte coquinaria composto per lo egregio maestro Martino coquo olim del Reverendissimo Monsignor Camorlengo et Patriarcha de Aquileia " (E. FAccioli éd., Arte della cucina. Libri di ricette, testi sopra lo scalco, il trinciante e i vini dal XIV al XIX secolo, t. I, Milan, 1966, p. 119).

7. L'identification avec Marin Grimani dans les catalogues Firmin-Didot et Pichon repose sur le titre quelque peu différent que transmet le manuscrit possédé par ces collectionneurs : «Libro de arte coquinaria edito per lo egregio e peritissimo maestro Martino coquo del Reverendissimo S. Cardinale de Aquileia ». Il n'y est donc fait pas mention du titre de camérier qui eût exclu Grimani - lequel ne fut d'ailleurs jamais que coadjuteur d'Aquilée.

8. Il n'y a de ce point de vue rien à tirer du ms. de la Biblioteca Apostolica Vaticana [désormais BAV], Urb. lat. 1203 [abrégé en V], dont j'ai signalé l'existence dès 1988 (B. Laurioux, « Le Registre de cuisine de Jean de Bockenheim, cuisinier du pape Martin V », dans Mélanges de l'École française de Rome. Moyen Âge, 100, 1988, p. 719, n. 59) et qui a été depuis publié par C. Benporat, Cucina italiana del Quattrocento, Florence 1996, p. 79-155 : en effet, cet exemplaire ne comporte pas de page de titre et c'est seulement au xvi ${ }^{\mathrm{e}}$ siècle qu'on l'a dénommé Epulario, peut-être parce qu'un lecteur avait reconnu la parenté du recueil avec celui qui fut publié en 1517 sous ce titre - et qui reprenait effectivement le Libro de arte coquinaria.

9. Plus précisément chez Christie's, pour une vente tenue à Londres les 13 et 14 novembre 1974 : Printed Books, Manuscripts \& Autograph Letters, Christie, Manson \& Woods, p. 89 et pl. 1.

10. Catalogue des livres précieux de M. A. Firmin-Didot, vente de 1883, Paris, 1883, p. 49, n 33 ; Livres rares et précieux manuscrits et imprimés, Catalogue de la Bibliothèque de feu M. le baron Jérôme Pichon, Paris, 1897, p. 170, no 591. Le même manuscrit formait le lot $n^{\circ} 411$ de la vente du 27 juin 1898 chez Sotheby's (signalé par M. E. Milham, "The manuscripts of Platina's De honesta voluptate ", Scriptorium, 26, 1972, p. 129).

11. Dans une lettre du 22 février 1994, le défunt libraire milanais Alberto Chiesa, qui détint ce manuscrit pendant des années, m'avait confié ne plus l'avoir en sa possession.

12. Puisque le olim de $W$ n'y figure pas.

13. Reproduit en facsimilé par A. Bertoluzza, Libro di cucina del maestro Martino de Rossi, Trente, 1993 (De coquina, vol. 3), puis édité par Benporat, op. cit., p. 157-231.

14. L'un en tête de la table: "Magistri Martini de Rubeys coqui Illustrissimi domini domini Ioanni Iacobi Trivultii de modo et ordine observandi in coquina. » L'autre précède le texte des recettes : «Libro de cosina composto et ordinato per lo egregio homo magistro Martino di Rossi della valle de Bregna Mediolanensis diocessis descenduto de la villa de Turre nato della casa de Sancto Martino Vidualis coquo dell Illustrisime signore Joanni Iacobo Trivultio etc. expertissimo in questa arte et como legeray prudentissimo. »

15. L'hypothèse selon laquelle Martino de' Rossi, auteur d'un Libro de cosina, et Maestro Martino, auteur du Libro de arte coquinaria, seraient deux personnes différentes, n'est pas soutenable: outre que la probabilité que deux homonymes signent le même texte paraît assez faible, elle est formellement démentie par la mention «Martino rubro Mediolanensi » figurant dans le manuscrit du De honesta voluptate revu par Platina et qui servit à l'editio princeps (Paris, BnF, lat. $\left.13003, \mathrm{f}^{\circ} 16 \mathrm{v}^{\circ}\right)$. Cf. M. G. Blasio, «Il De honesta voluptate et valitudine di Bartolomeo Platina », RR. Roma nel Rinascimento, 1999, p. 14-20. 
16. Né en 1440, Trivulzio a été élevé en même temps que Galeazzo Maria Sforza, devenu duc de Milan en 1466. Dès l'année suivante, il se voit confier sa première compagnie.

17. Je m'appuie ici sur les données rassemblées par C.Benporat, «Un frammento inedito di Maestro Martino », Appunti di Gastronomia, 26, giugno 1998, p. 109-121.

18. Benporat, ibid., p. 117, a fait toutefois remarquer que le menu en question est étrangement identique à celui du festin des noces du «comte Jeronimo» contenu dans un manuscrit manifestement méridional (New York, Pierpont Morgan Library, ms. Bühler 19; éd. Benporat, Cucina italiana del Quattrocento, op. cit., p. 282-284).

19. G. Chiesi, «Martino Rossi : un cuoco bleniese alla corte ducale ", dans Bertoluzza, op. cit., p. 11-15. Malheureusement, cet archiviste ne publie pas intégralement les documents en question et n'en fournit même pas un régeste en bonne et due forme. Je resterai donc prudent quant à leur interprétation.

20. La plupart des documents cités ibid., n. 14, concernant la récupération de créances dans cette même vallée, on est tenté de les attribuer au même bénéficiaire : mais, ici comme ailleurs, les débiteurs récalcitrants ne devaient pas être rares!

21. BenPorAt, Cucina italiana del Quattrocento, op. cit., p. 24.

22. L'idée de cette enquête m'a été donnée par le prudent rapprochement qu'a fait C. Märtl entre le Martino qu'elle rencontrait dans les comptes de menues dépenses et l'auteur du Libro de arte coquinaria (« Humanistische Kochkunst und kuriale Ernährungsgewohnheiten um die Mitte des 15. Jahrhunderts", dans Herrschaft und Kirche im Mittelalter, Gedenksymposium zum ersten Todestag von Norbert Kamp, Braunschweig, 2001, p. 57-58). Les quelques pièces supplémentaires que j'ai engrangées confirment pleinement, me semble-t-il, l'intuition de cette historienne.

23. Cuisinier du commun du pape Martin V : cf. B. Laurioux, «Le Registre de cuisine de Jean de Bockenheim, cuisinier du pape Martin V », Mélanges de l'École française de Rome. Moyen Âge, 100, 1988, p. 709-760.

24. Le document $\mathrm{n}^{\circ} 2$ qui concerne un Martino cuisinier du commun pose évidemment problème : s'agit-il d'un homonyme ou d'une erreur de copiste?

25. Le compte du cubicularius secretus Heinrich Dailman mentionne, outre un « Magistro Petro coco secreto » $\left(\mathrm{n}^{\circ} \mathrm{7}\right)$, un « Johanne Grotte [ou Groot comme indiqué dans la marge] coco secreto infirmo » qui reçoit un don de 6 ducats le 30.XI.1469 (Archivio di Stato di Roma [désormais abrégé en ASR], Camerale I, 1481, fo $\left.28 \mathrm{r}^{\circ}\right)$.

26. Cf. le document $n^{\circ} 7$, où Martino reçoit une gratification de 10 ducats, contre seulement 8 à son collègue Maestro Pietro et 6 à Giovanni qui doit être leur aide « in coquina secreta ».

27. Comme les moules de bois pour réaliser les raviolis de la collation $\left(n^{\circ} 3\right)$.

28. ASR, Camerale I, 1479, fo $116 \mathrm{r}^{\circ}$. Un « Maestro Martino » est aussi cité en décembre 1462 dans le compte de Tesoreria Segreta de Pie II (ASR, Camerale I, 1289, $\mathrm{f}^{\circ} 56 \mathrm{v}^{\circ}$ ) pour des achats de literie.

29. Doc. $\mathrm{n}^{\circ} 1$.

30. Märtl, loc. cit., p. 57.

31. Ibid.

32. Avancé par le rédacteur du catalogue Christie's de 1974, ce rapprochement est largement confirmé par l'analyse paléographique que l'on peut faire de la planche accompagnant ce catalogue : $1^{\circ}$ les $e$ prolongés d'un trait et la panse des $g$ sont très proches de l'écriture cursive de Sanvito, notamment dans le Juvénal de la Laurentiana (J. Wardrop, The Script of Humanism. Some Aspects of Humanistic Script, 1460-1560, Oxford, 1963, pl. 27) et les capitales (A penché, barre inférieure du $E$ et du $L$ remontante, jambage gauche du $M$ plus court que le droit) sont identiques à celles qui figurent dans ce même manuscrit; $2^{\circ}$ la forme caractéristique du $C$ majuscule, encadrant la lettre suivante, se retrouve par exemple dans le Cicéron de Londres (Ibid., pl. 28); $3^{\circ}$ le seul doute pourrait venir des ligatures $c t$ et $s t$, nettement moins exagérées dans le ms. que dans les productions habituelles de Sanvito, dont c'est la marque typique ; $4^{\circ}$ mais je constate qu'au bout de la $4^{\mathrm{e}}$ ligne du titre est dessinée une petite feuille renversée, que l'on retrouve dans 
le ms. autographe de Sanvito donné à Monselice (Ibid., pl. 21) et sur la page de titre du Cicéron d'Eton College.

33. Märtl, loc. cit., p. 56.

34. C'est ce que Platina affirme dans le De principe, en 1471 : « Me adolescentem et quadriennio militem levis armaturae fuisse, et partim sub Francisco Sfortia, partim sub Nicolao Picinino egregiis copiarum Ducibus militasse vidisseque multa quae ad hanc disciplinam pertinent. » (BAV, ms. Vat. Lat. $3814, \mathrm{f}^{\circ} 3 \mathrm{r}^{\circ}$.)

35. Le condottiere Niccolò Piccinino, sous les ordres duquel Platina prétend également avoir servi, étant mort en 1444.

36. Le seul acte milanais précédant 1450 ne paraît pas mentionner la qualité de cuisinier ducal pour Martino Rossi (Chiesi, loc. cit., p. 13).

37. En 1461, le mandement de la duchesse Bianca Maria cité supra le qualifie de coquus noster.

38. Cf. surtout le poème 124 (Ad Lutium de Caluo degenere) des Élégies de Callimachus, alias Filippo Buonaccorsi. Ces arguments sont plus amplement développés dans Autour du De Honesta Voluptate de Platina. Gastronomie, humanisme et société à Rome au milieu du $x v^{e}$ siècle sous presse dans la collection Micrologus'Library.

39. Même si pendant quelques semaines il fut gardé à résidence au palais du Vatican, avant d'être définitivement libéré en 1469.

40. Abréviations : ASR $=$ Archivio di Stato di Roma ASV $=$ Archivio Segreto Vaticano ; duc. $=$ ducati ; bol. = bolognini.

41. Marco Barbo, cardinal prêtre de Saint-Marc, qui a remplacé Ludovico Trevisan comme camérier.

42. Cette mention figure en marge de la plupart des notices du compte (avec quelquefois le R. étendu en Regas.) d'une encre différente : elle a été ajoutée postérieurement comme preuve de contrôle.

43. Le même jour, sont distrbués 10 duc. à un cubicularius, 6 à Michael le forerius ; le 9 décembre, 5 duc. à Baldewinus crédencier secret, infirmo.

\section{RÉSUMÉS}

Bien connu pour avoir été l'inspirateur du versant culinaire du De honesta voluptate de Platina, Maestro Martino a vu son existence et sa carrière progressivement dévoilées au gré de la réapparition des manuscrits de son livre de recettes, le Libro de arte coquinaria. Les notations contenues dans les archives de la papauté, et notamment dans les comptes de menues dépenses de la maison pontificale, permettent de compléter son parcours avec un long séjour à Rome, au service des papes Paul II puis Sixte IV. Cuisinier secret engagé par le pape avant même la mort de son précédent patron (le patriarche d'Aquilée), Maestro Martino a joué un rôle de premier plan dans la préparation de la collation du carnaval de 1466, un des temps forts du pontificat de Paul II.

Cocus Secretus of the Pope. Well known for having been the inspiration of the section on cooking in the De honesta voluptate of Platina, Maestro Martino's existence and career have been progressively revealed with the reappearance of the manuscripts of his book of recipes, the Libro de arte coquinaria. The notes contained in the archives of the papacy, notably the accounts of the pontifical household, allow us to complete what we know of his career by a prolonged stay in 
Rome in the service of Paul II and Sixtus IV. A secret cook, engaged even before the death of his previous patron, the patriarch of Aquilea, Maestro Martino played a leading role in the preparation of the collation for the carnival of 1466, a highlight of the pontificate of Paul II.

INDEX

Keywords : cooks, household accounts, humanism, Paul II, popes

Mots-clés : comptes d'approvisionnement, cuisiniers, humanisme, papes, Paul II

\section{AUTEUR}

\section{BRUNO LAURIOUX}

Université Versailles-Saint-Quentin-en-Yvelines, UFR des Sciences sociales et des humanités, 47, bd Vauban, F-78047 Guyancourt 